\title{
Comparative Evaluation of Anoxomat and Conventional Anaerobic GasPak J ar Systems for the Isolation of Anaerobic Bacteria
}

\author{
May Shahin Wafaa J amal Tina Verghese V.O. Rotimi \\ Departments of Microbiology, Faculty of Medicine, Kuwait University and Mubarak Al-Kabeer Teaching Hospital, \\ Kuwait
}

\section{Key Words}

Anoxomat · GasPak jar system - Anaerobes, isolation

\begin{abstract}
Objectives: To evaluate the performance of the Anoxomat, in comparison with the conventional anaerobic GasPak jar system, for the isolation of obligate anaerobes. Method: Anoxomat, model WS800, and anaerobic GasPak jar system (Oxoid) were evaluated. Anoxomat system utilized a gas mixture of $80 \% \mathrm{~N}_{2}, 10 \% \mathrm{CO}_{2}$ and $10 \% \mathrm{H}_{2}$, while the GasPak used a gas mixture of $90 \% \mathrm{H}_{2}$ and $10 \% \mathrm{CO}_{2}$. An anaerobic indicator within the jars monitored anaerobiosis. A total of 227 obligate anaerobic bacteria comprising 116 stock strains, 5 ATCC reference strains and 106 fresh strains, representing different genera, were investigated for growth on anaerobic agar plates and scored for density, colony sizes, susceptibility zones of antibiotic inhibition and the speed of anaerobiosis (reducing the indicator). Results: The results demonstrate that the growth of anaerobic bacteria is faster inside the Anoxomat jar than in the anaerobic GasPakjar system. Of the 227 strains tested, the colonies of 152 $(67 \%)$ were larger (by size range of $0.2-2.4 \mathrm{~mm}$ ) in the Anoxomat at $48 \mathrm{~h}$ than in the GasPak jar compared with only $21 \%$ (range $0.1-0.3 \mathrm{~mm}$ ) that were larger in the GasPak than in the Anoxomat. The remaining $12 \%$ were
\end{abstract}

equal in their sizes. There was no measurable difference in the colony sizes of the reference strains. The Porphyromonas asaccharolytica strains failed to grow within the GasPak system but grew inside the Anoxomat. With the Anoxomat, anaerobiosis was achieved about 35 min faster than in the GasPak system. The density of growth recorded for 177 (78\%) strains was heavier in the Anoxomat than in the GasPak jar. The zones of inhibition of the antibiotics tested were not different in the two systems. Conclusion: The Anoxomat system provided superior growth, in terms of density and colony size, and achieved anaerobiosis more rapidly. Evidently, the Anoxomat method is more reliable and appears to support the growth of strict anaerobes better.

Copyright @2003S. Karger AG, Basel

\section{Introduction}

Anaerobes belong to a group of microorganisms that are usually difficult to culture in many routine diagnostic laboratories. Anaerobic methods used for their isolation include anaerobic jars, plastic anaerobic bags, the PRAS (prereduced anaerobically sterilized) method and the anaerobic chamber. The last two techniques are rather timeconsuming, require complex or expensive equipment, and utilize PRAS media. They are essential for work with

\begin{tabular}{ll}
\hline KARGER & ( 2003 S. Karger AG, Basel \\
Fax +4161306 1234 & 1011-7571/03/0122-0081\$19.50/0 \\
$\begin{array}{l}\text { E-Mail karger@karger.ch } \\
\text { www.karger.com }\end{array}$ & $\begin{array}{l}\text { Accessible online at: } \\
\text { www.karger.com/mpp }\end{array}$
\end{tabular}

Prof. V.O. Rotimi

Department of Microbiology, Faculty of Medicine

Kuwait University, PO Box 24923

13110 Safat (Kuwait)

Tel./Fax +965 533 2719, E-Mail vincent@hsc.kuniv.edu.kw 


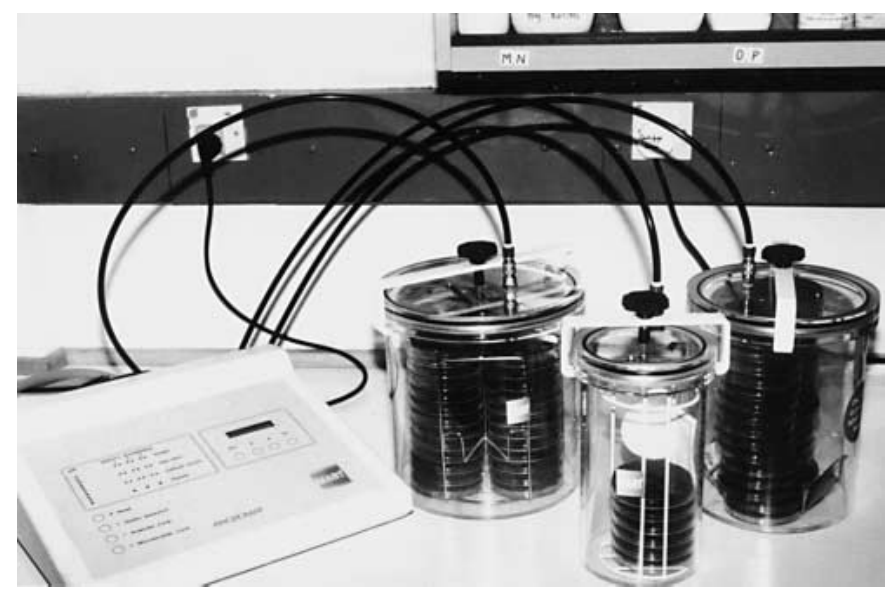

Fig. 1. Anoxomat machine.

anaerobes of the normal flora as these organisms are often more oxygen-sensitive than clinical isolates and as such are not really required in a routine clinical laboratory.

In the last two decades, comparative studies have shown that when clinical samples are collected, transported and processed properly, recovery of clinically significant anaerobes is as good with jars as with more complex methods [1]. To obtain a reliable result with any anaerobic jar there must be adequate replacement of the oxygenated environment with an anaerobic atmosphere. This is usually achieved by introducing a gas mixture containing $\mathrm{H}_{2}$ into the jar. In the presence of catalyst, the oxygen combines with $\mathrm{H}_{2}$ to produce water; as a result an anaerobic environment is established. A gas generator envelope that releases $\mathrm{H}_{2}$ and $\mathrm{CO}_{2}$ when water is added is commercially available and many clinical laboratories have found the GasPak jar method particularly convenient. However, the jar must remain inviolate for at least $48 \mathrm{~h}$ after the GasPak has been activated [2]. On the other hand, an 'evacuation replacement' system may be used [3]. Air is removed from the sealed jar by repeatedly drawing a vacuum and then filling the jar with anaerobic gas mixture. The Anoxomat (Mart Microbiology BV, Lichtenvoorde, Holland) uses an automated evacuation replacement technique to create an anaerobic or microaerophilic environment in the jar. The anaerobic program repeats the evacuation-replacement cycle 3 times. During each cycle, the anaerobic gas mixture replaces $80 \%$ of the jar content. After the third cycle, any remaining oxygen and any oxygen liberated from the media in plates is removed by combination with $\mathrm{H}_{2}$, a reaction made possible by a palladium catalyst. This system also provides

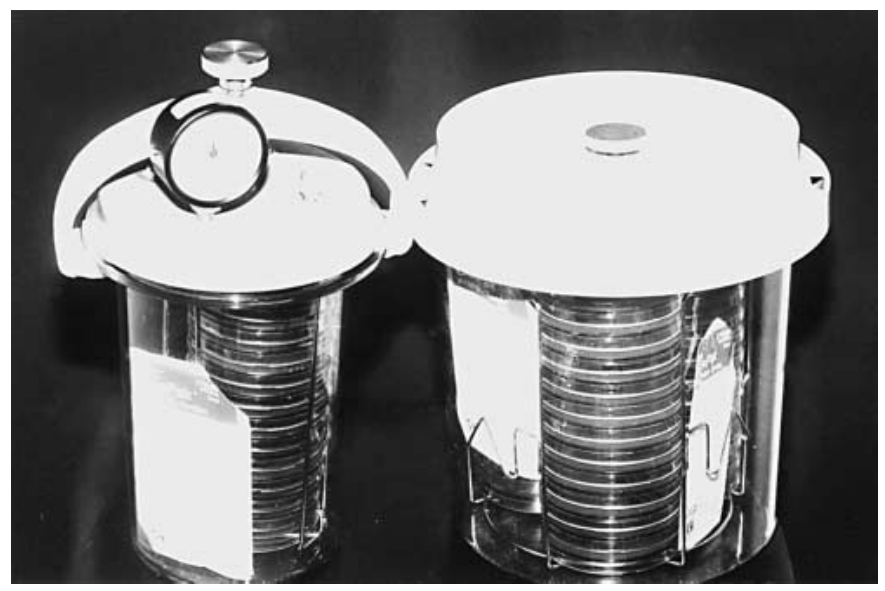

Fig. 2. GasPak jar system.

internal quality assurance programs that perform additional tests, when selected, such as a check for leaks, a test for catalyst activity, and a test for sufficient gas supply. An added advantage is that the Anoxomat system can also be used for the isolation of microaerophilic and capnophilic organisms.

The purpose of this study was to evaluate the capability of the Anoxomat to support the growth of obligate anaerobes, the performance of antibiotic susceptibility testing (AST) and speed of achieving anaerobiosis within the Anoxomat jar. The performance of the Anoxomat would then be compared with that of an existing anaerobic GasPak jar system used in our Anaerobe Reference/ Research Laboratory situated in the Faculty of Medicine, Kuwait University.

\section{Materials and Methods}

\section{Anaerobic Techniques}

Two pieces of anaerobic apparatus were used in this study: the Anoxomat model WS800, which has connections for three jars (MART Microbiology BV, Lichtenvoorde, Netherlands, fig. 1), and the GasPak jar system using anaerobic jars (Oxoid, Basingstoke, England) and gas generating envelopes (Oxoid), which release $\mathrm{H}_{2}$ and $\mathrm{CO}_{2}$ when water is added (fig. 2). The Gas Generating Kit system is a reliable and convenient method for producing anaerobic conditions in standard anaerobic jars containing catalysts and is available in many routine diagnostic microbiology laboratories. Palladium catalysts were placed inside both systems to catalyze the reaction between oxygen and hydrogen. A gas mixture of $80 \% \mathrm{~N}_{2}, 10 \% \mathrm{H}_{2}, 10 \% \mathrm{CO}_{2}$, supplied by a gas cylinder, was the replacement gas used inside the Anoxomat. An anaerobic indicator and a plate of Pseudomonas aeruginosa were placed inside the jars to monitor anaerobiosis. 
The Anoxomat Cycles

In order to set up the Anoxomat system (fig. 1) a jar is attached to the Anoxomat machine by a tube which forms a gas-tight fit by means of a snap-shut coupling adaptor on the lid. The vacuum pump is connected to the Anoxomat with a high-pressure tube and then the Anoxomat is connected to the gas cylinder by another high-pressure tube. On pressing the Anaerobic Cycle select button, the jar is evacuated a preselected number of times (in our experience, 3 cycles with a total cycle time of about $70 \mathrm{~s}$ is sufficient) to -0.8 bar and then the partial vacuum is replaced with the gas mixture to a positive pressure of +0.03 bar, after which a sound signals the completion of the cycle. Illumination of green light-emitting diodes indicates when all conditions are satisfactory.

\section{Bacterial Strains and Growth Conditions}

Two hundred and twenty-seven strains of obligate anaerobic bacteria representing 6 different genera (Bacteroides, Clostridium, Fusobacterium, Prevotella, Porphyromonas and Peptostreptococcus) of clinical significance were tested. This panel was made up of 116 stock isolates (obtained from patients in Mubarak Hospital, Kuwait), 106 consecutively collected fresh clinical isolates from our clinical microbiology laboratory (single-patient isolates) and 5 ATCC reference strains (Bacteroides fragilis ATCC 25285, Baceroides thetaiotaomicron ATCC 29741, Peptostreptococcus anaerobius ATCC 27337, Clostridium perfringens ATCC 13124 and Clostridium difficile ATCC 43593). The stock organisms had been kept frozen at $-80^{\circ} \mathrm{C}$ as heavy suspensions in brain-heart infusion broth (Oxoid), with $10 \%$ glycerol as preservative. Frozen strain suspensions were allowed to thaw to room temperature in an anaerobic cabinet, mixed thoroughly and subcultured twice on Brucella agar (Oxoid), containing $6 \%$ lyzed horse blood, to check the purity of the cultures.

\section{Inoculum Preparation and Incubation Methods}

Suspensions were prepared by emulsifying bacterial isolates in thioglycolate broth (Oxoid) to the equivalent of 2.0 McFarland turbidity standard $\left(10^{7} \mathrm{cfu} / \mathrm{ml}\right)$. The same suspension was used for growth assessment and AST in both systems. Ten microliters of each suspension was inoculated on duplicate plates of Brucella blood agar (all the plates were poured from the same batch of media, to the same depth and dried to the same degree) and streaked by a standard plating method [4] that allowed isolation of single colonies and semiquantification (density of growth) after incubation [5]. One plate of each pair was placed in a GasPak anaerobic jar. A GasPak gas generator envelope, to which $10 \mathrm{ml}$ of water was added, was immediately placed in an upright position inside the anaerobic jar and the lid closed. The other plate was placed in the Anoxomat jar. This jar was attached to the Anoxomat machine and the anaerobic cycles completed. Both jars contained palladium catalyst and were incubated in a $37^{\circ} \mathrm{C}$ incubator for $48 \mathrm{~h}$.

\section{AST with Anoxomat and GasPak Systems}

Thirty isolates of $B$. fragilis, 10 of $C$. difficile, 20 of $C$. perfringens and 20 of Prevotella bivia were tested for their susceptibility to amoxicillin-clavulanic acid, clindamycin, imipenem, metronidazole and penicillin by a disk diffusion method using both anaerobic incubation techniques. The strain was grown in Brucella broth for $48 \mathrm{~h}$ and then adjusted to $0.5 \mathrm{McF}$ arland turbidity standard suspension in thioglycolate broth. An inoculum of $10 \mu \mathrm{l}$ of the suspension was carefully spread on a Brucella agar plate in three different directions using a sterile glass rod. The antibiotic disks were placed on the sur- face of the agar equidistant from each other and then one plate was placed in the GasPak jar and the other in the Anoxomat jar. Included in each run, as controls, were reference strains of $B$. fragilis ATCC 25285 and $C$. perfringens ATCC 13124 . The two jars were incubated in a $37^{\circ} \mathrm{C}$ incubator for $48 \mathrm{~h}$ after which the zones of inhibition around each antibiotic disks were measured and interpreted as resistant or sensitive according to the interpretation criteria recommended by the NCCLS [6].

\section{Time Required to Achieve Anaerobiosis}

The speed of achieving anaerobiosis was recorded by observing the time it took for the chemical anaerobic indicator (Oxoid) included in each jar to change color from pink to white. Growth of $P$. aeruginosa on the nutrient agar plate after 48-hour incubation was taken to mean that anaerobiosis was not achieved and that there had been jar failure.

\section{Results}

Table 1 shows the performance of both Anoxomat jar and anaerobic GasPak jar systems in terms of growth and colony sizes. Of the total 222 stock and fresh strains tested, $152(68.5 \%)$ were larger (by a range of $0.2-$ $2.4 \mathrm{~mm}$ ) in the Anoxomat at $48 \mathrm{~h}$ incubation than in the GasPak jar. Only 48 (21.6\%) produced slightly bigger colonies (range 0.1-0.3 mm) in the GasPak than Anoxomat and the remaining $27(12.2 \%)$ strains gave colonies that were equal in size. These differences are presented graphically in figure 3 . However, differences in the colony sizes of the reference strains in both systems were negligible. Several strains of Bacteroides vulgatus, Clostridium sporogenes and C. difficile, in particular, produced 3 to 5-fold larger colonies in the Anoxomat system than in the GasPak system. Six strains of Porphyromonas asaccharolytica failed to grow inside the GasPak anaerobic jars but grew inside the Anoxomat jar after $48 \mathrm{~h}$. This discrepancy was partially resolved by extended incubation, for another $72 \mathrm{~h}$, when 4 out of 6 strains grew in the GasPak jar. These results demonstrate that the growth of many anaerobic bacteria is faster inside the Anoxomat jars than the GasPak anaerobic jars. All the reference strains grew to more or less the same colony sizes in both systems. Analysis by density of growth showed that $177(78 \%)$ of the strains produced heavier growth density (++++) in the Anoxomat system than in the GasPak jar (+++) (data not shown). The chemical anaerobic indicator consistently became decolorized faster, by $35-38 \mathrm{~min}$, in the Anoxomat jars than in GasPak jars. Achievement of anaerobiosis in all jars was further demonstrated by the lack of growth of $P$. aeruginosa included in each jar.

The results of the AST, measured by the zones of inhibition around each disk, generated by incubation in the 
Fig. 3. Comparison of mean colony sizes in the GasPak anaerobic jar ( $(\mathbf{)}$ ) and Anoxomat jar $(\square)$. The differences in colony sizes were most apparent with $C$. sporogenes $(3 \mathrm{~mm})$, C. difficile $(1.7 \mathrm{~mm})$ and $B$. vulgatus (2 mm).

Table 1. Performance of GasPak jar and Anoxomat jar in the isolation of anaerobic organisms measured by comparative colony sizes after 48-hour incubation

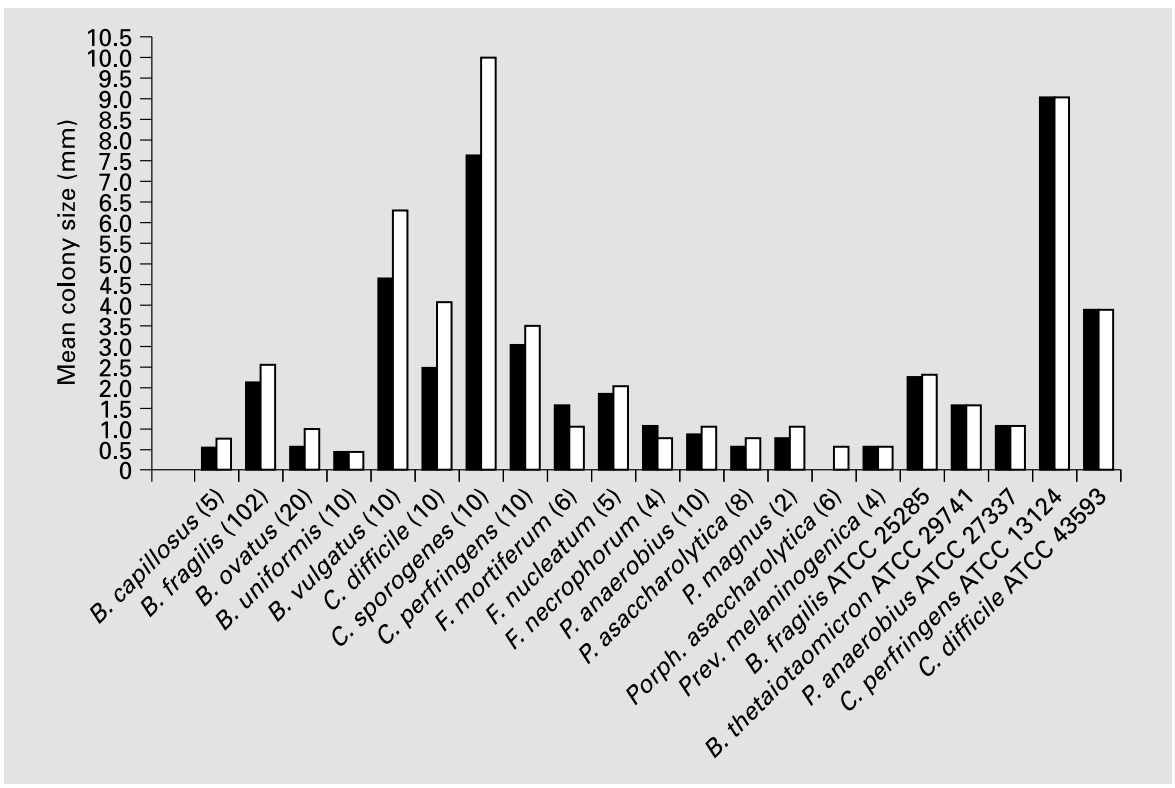

\begin{tabular}{lrll}
\hline Organism & Isolates & \multicolumn{2}{l}{ Mean colony size, mm } \\
\cline { 2 - 4 } & & GasPak jar & Anoxomat jar \\
\hline Bacteroides capillosus & 5 & 0.5 & 0.75 \\
Bacteroides fragilis & 102 & 2.1 & 2.5 \\
Bacteroides ovatus & 20 & 0.5 & 0.95 \\
Bacteroides uniformis & 10 & 0.36 & 0.36 \\
Bacteroides vulgatus & 10 & 4.6 & 6.3 \\
Clostridium difficile & 10 & 2.4 & 4 \\
Clostridium sporogenes & 10 & 7.6 & 10 \\
Clostridium perfringens & 10 & 3 & 3.5 \\
Fusobacterium mortiferum & 6 & 1.5 & 1 \\
Fusobacterium nucleatum & 5 & 1.8 & 2 \\
Fusobacterium necrophorum & 4 & 1 & 0.7 \\
Peptostreptococcus anaerobius & 10 & 0.8 & 1 \\
Peptostreptococcus asaccharolytica & 8 & 0.5 & 0.7 \\
Peptostreptococcus magnus & 2 & 0.7 & 1 \\
Porphyromonas asaccharolytica & 6 & 0 & 0.5 \\
Prevotella melaninogenica & 4 & 0.5 & 0.5 \\
Control strains & 5 & & \\
$\quad$ Bacteroides fragilis ATCC 25285 & 1 & 2.2 & 2.3 \\
Baceroides thetaiotaomicron ATCC 29741 & 1 & 1.5 & 1.6 \\
Peptostreptococcus anaerobius ATCC 27337 & 1 & 1 & 1 \\
Clostridium perfringens ATCC 13124 & 1 & 9 & 9 \\
Clostridium difficile ATCC 43593 & 1 & 3.8 & 3.9 \\
& & &
\end{tabular}

Anoxomat system are compared with those of the GasPak system in table 2 and graphically in figure 4 . A susceptible category was assigned to each zone of inhibition based on the current NCCLS cutoff criteria. There was general agreement within $1 \mathrm{~mm}$ from the reference strain results.
No observable difference was apparent in the two systems but the diameter of the zones of inhibition around the metronidazole disk was generally larger in the Anoxomat system. However, there were no results that generated discrepancies with susceptibility interpretation. 
Fig. 4. A demonstration of the differences in the zone of inhibition around the 5 antibiotic disks for each of the organisms. $\mathbb{\nabla}=C$. difficile $; \square=$ Prev. bivia $;$ 目 = C. perfringens $;=$ B. fragilis. $\mathrm{P}=$ Penicillin; $\mathrm{CC}=$ clindamycin; $\mathrm{AMC}=$ amoxicillin/clavulanate; IMP $=$ imipenem; MET $=$ metronidazole .

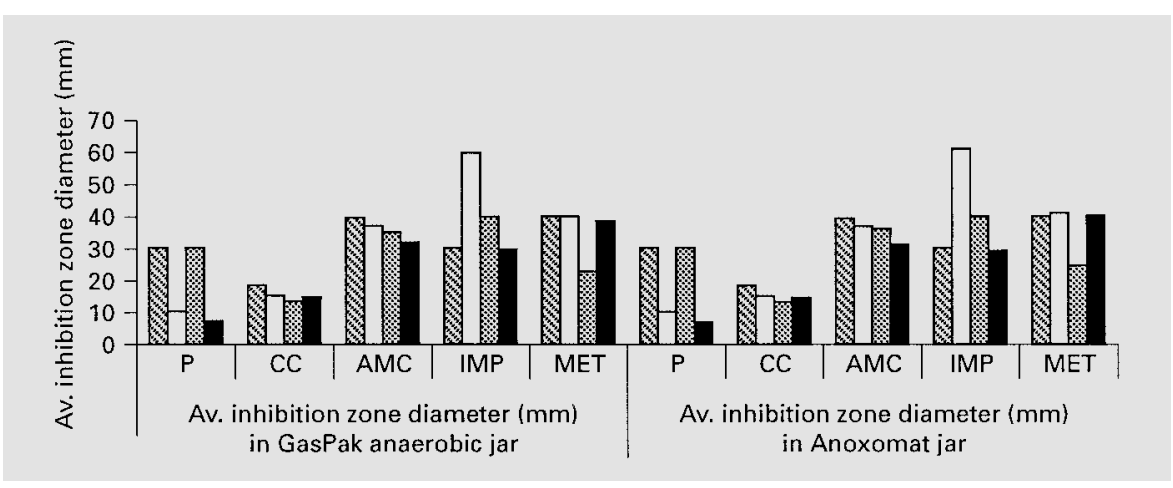

Table 2. A comparison of the average inhibition zone diameter $(\mathrm{mm})$ of anaerobic organisms after $48 \mathrm{~h}$ of incubation

\begin{tabular}{|c|c|c|c|c|c|c|c|c|c|c|}
\hline \multirow[t]{3}{*}{ Organisms } & \multicolumn{10}{|c|}{ Average inhibition zone diameter, $\mathrm{mm}$} \\
\hline & \multicolumn{5}{|c|}{ GasPak anaerobic jar } & \multicolumn{5}{|c|}{ Anoxomat jar } \\
\hline & $\mathrm{P}$ & $\mathrm{CC}$ & $\mathrm{AMC}$ & IMP & MET & $\mathrm{P}$ & $\mathrm{CC}$ & AMC & IMP & MET \\
\hline Clostridium difficile & 30 & 18.3 & 39.3 & 30 & 40 & 30 & 18.3 & 39.3 & 30 & 40 \\
\hline Prevotella bivia & 10 & 15 & 37 & 60 & 40 & 10 & 15 & 37 & 61 & 41.1 \\
\hline Clostridium perfringens & 30 & 13 & 35 & 40 & 23 & 30 & 13 & 36 & 40 & 24.5 \\
\hline Bacteroides fragilis & 6.8 & 14.5 & 31.5 & 29.5 & 38.5 & 6.8 & 14.5 & 31.5 & 29.3 & 40.5 \\
\hline
\end{tabular}

$\mathrm{P}=$ Penicillin $; \mathrm{CC}=$ clindamycin $; \mathrm{AMC}=$ amoxicillin/clavulanate $\mathrm{IMP}=$ imipenem $; \mathrm{MET}=$ metronidazole.

\section{Discussion}

The ability of the Anoxomat to effectively support the growth of clinically significant gram-positive and gramnegative anaerobic organisms, as well as microaerophilic and capnophilic bacteria, was first assessed by Brazier and Smith [7], who showed superiority of the Anoxomat system over anaerobic cabinets in 52\% of cases. Because Anoxomat utilizes an evacuation-replacement technique, this observation was contrary to the previously held position that anaerobic cabinets are superior to the jar system in terms of internally generated gases and the single evacuation replacement method, resulting in denser growth and higher yield of anaerobes [8-10]. However, the results of our study support a previous finding [7] and show that the Anoxomat is superior to the GasPak system in $67 \%$ of the cases. In terms of AST, which is a crucial task for any clinical microbiology laboratory, no serious discrepancies in results were obtained with respect to the zone sizes and correct interpretation. Larger zones of inhibition around metronidazole disks observed with the Anoxomat system support a superior anaerobiosis within the jar.
Since the factors affecting colony size were standardized, the only variable factor between the two systems was the speed of achieving anaerobiosis. In our study, the average time for achieving anaerobiosis in the Anoxomat system was 35 min faster than in the GasPak system, as judged by the time taken for discoloration of the anaerobic indicator. Conceivably, this difference could allow the anaerobes to enter into the exponential phase of growth faster and hence produce larger colonies in the Anoxomat system after 48-hour incubation. Our observation is supported by the findings of Imhof and Heinzer [11], who compared seven commercial systems, including the GasPak and evacuation-replacement systems, for their ability to decrease oxygen concentration in an anaerobic jar. These authors found that the evacuation-replacement system was the most rapid and reliable method for generation of an anaerobic environment.

In this study, we observed no jar failure with either the Anoxomat or the GasPak systems, an observation supported by a previous report [11] but slightly discordant with that of Miller et al. [12], who reported a failure rate of $10 \%$ with the BBL GasPak system. Even though the 
Oxoid GasPak system did not yield growth of Porph. asaccharolytica at $48 \mathrm{~h}$, extended incubation for another $72 \mathrm{~h}$ produced growth of a high proportion of the strains tested in this study. It is noteworthy that this organism is very sensitive to oxygen and therefore it is conceivable that the slower rate of achieving anaerobiosis in the GasPak system might have affected its generation time and hence produced a requirement for extended incubation.

The Anoxomat system has some compelling features, which make it attractive for use in a routine clinical laboratory. It is very easy to use and evacuation 3 times removes virtually all the oxygen within the jar with the result that the function of the palladium catalyst is limited to removing the residual oxygen. In addition, this system is versatile in that it can also be used to culture microaerophilic and capnophilic organisms [7]. Apart from the initial cost, the savings on $\mathrm{CO}_{2}$ incubator, gas generating kits and pure $\mathrm{CO}_{2}$ supplies make the equipment cost-effective. However, the relative merit of the GasPak system still stands. It is less bulky and takes up little bench space.

In conclusion, the results of this study indicate that the Anoxomat system, an evacuation-replacement system, is a more rapid and reliable system for isolation of obligate anaerobes including very oxygen-sensitive species. Its use in the clinical microbiology laboratory may enhance recovery of anaerobes from clinical specimens and its versatility is a strong point in favor.

\section{Acknowledgment}

This study was partly supported by Kuwait University Research Grant No. MI 110.

\section{References}

1 Citron DM: Specimen collection and transport, anaerobic culture techniques and identification of anaerobes. Rev Infect Dis 1984;6(suppl):5155.

2 Bartlett JG: Anaerobic bacteria; in Gorbach SL, Bartlett JG, Blacklow NR (eds): Infectious Diseases. Philadelphia, Saunders, 1992, pp 1555-1567.

3 Fildes P, McIntosh J: An improved form of anaerobic jar. Br J Exp Pathol 1921;2:153.

4 Gillies RR, Dodds TC: Bacteriology Illustrated. Edinburgh, Churchill Livingstone, 1976.
5 Rotimi VO, Duerden BI: The development of bacterial flora in normal neonates. $\mathrm{J}$ Med Microbiol 1981;14:51-62.

6 National Committee for Clinical Laboratory Standards: Methods for Antimicrobial Susceptibility Testing for Anaerobic Bacteria. Approved Standard M11-A4, vol 17, No. 22. Villanova, NCCLS, 1997.

7 Brazier JS, Smith SA: Evaluation of the Anoxomat: A new technique for anaerobic and microaerophilic clinical bacteriology. J Clin $\mathrm{Pa}-$ thol 1989;42:640-644.

8 Rosebury T, Reynolds JB: Continuous anaerobiosis for cultivation of spirochaetes. Proc Soc Exp Biol Med 1964;117:813-815.
9 Drasar BS: Cultivation of anaerobic intestinal bacteria. J Pathol Bacteriol 1967;94:417-427.

10 Aranki A, Syed SA, Kenney EB, Freter R: Isolation of anaerobic bacteria from human gingival and mouse cecum by means of a simplified glove box procedure. Appl Microbiol 1969;17: 568-576.

11 Imhof A, Heinzer I: Continuous monitoring of oxygen concentrations in several systems for cultivation of anaerobic bacteria. J Clin Microbiol 1996;34:1646-1648.

12 Miller PH, Wiggs LS, Miller JM: Evaluation of AnaeroGen system for growth of anaerobic bacteria. J Clin Microbiol 1995;33:23882391. 